\title{
AVALIAÇÃO dO PROCESSO ADAPTATIVO DE UM IDOSO PORTADOR DE HIPERTENSÃo ARTERIAL ${ }^{1}$
}

\author{
Taciana Cavalcante de Oliveira ${ }^{2}$ \\ Thelma Leite de Araújo $^{3}$ \\ Elizabeth Mesquita Melo ${ }^{2}$ \\ Diva Teixeira de Almeida ${ }^{4}$
}

Oliveira TC, Araújo TL, Melo EM, Almeida DT. Avaliação do processo adaptativo de um idoso portador de hipertensão arterial. Rev. Latino-am Enfermagem 2002 julho-agosto; 10(4):530-6.

Objetivamos avaliar o processo adaptativo de um idoso portador de hipertensão arterial, identificar diagnósticos de enfermagem e implementar ações de enfermagem. As etapas do estudo foram desenvolvidas em domicílio, utilizando-se, para a coleta de dados, um instrumento baseado no modelo de Roy. Após avaliação de comportamentos e estímulos, elaboramos seis diagnósticos: mobilidade, andar e/ou coordenação restritos; deficiência de um sentido primário; privação do sono; baixa autoestima; transição de papel e solidão. Concluímos que as metas e intervenções implementadas de enfermagem foram importantes, já que permitiram, de maneira geral a substituição de respostas ineficazes por adaptativas.

DESCRITORES: idoso, hipertensão, comportamento, diagnóstico de enfermagem

\section{eVAlUATION Of THE ADAPTION PROCESS IN ELDERLY PEOPLe WITH HIGH BLOOD PRESSURE}

This study aimed at evaluating the adaption process of an elderly person with high blood pressure by establishing nursing diagnoses and implementing nursing actions. The phases of the study were developed in the patient's home and an instrument based on Roy's model was used data collection. After behaviors and stimuli were evaluated, six diagnoses were elaborated: restricted mobility, gait, and/or coordination; impairment of a primary sense; sleep deprivation; low self-esteem; role transition and loneliness. It was concluded that nursing goals and interventions were important once they allowed, in a general fashion, ineffective responses to be replaced for adaptive ones.

DESCRIPTORS: elderly, high blood pressure, behavior, nursing diagnosis

\section{eVAluación del proceso de adaptación de Un ANCIANo portador de HIPERTENSIÓN ARTERIAL}

El objetivo es evaluar el proceso de adaptación de un anciano portador de hipertensión arterial, establecer diagnósticos de enfermería y poner en práctica las acciones correspondientes. Las etapas del estudio fueron desarrolladas en el domicilio, utilizándose para la recoleccion de datos un instrumento basado en el modelo de Roy. Después de la evaluación de comportamientos y estímulos, elaboramos seis diagnósticos: dificultades para caminar, movilidad y/o coordinación restringidas; deficiencia de un sentido primario; privación del sueño; baja autoestima; cambios de rol e soledad. Concluimos que las metas e intervenciones de enfermería aplicadas fueron importantes, ya que, permitieron en general la substitución de respuestas ineficaces por adaptativas.

DESCRIPTORES: anciano, hipertensión, comportamiento, diagnóstico de enfermería

\footnotetext{
${ }^{1}$ Trabalho desenvolvido na disciplina Enfermagem Clínico-Cirúrgica do Programa de Pós-Graduação em Enfermagem da Universidade Federal do Ceará. Inserido no Projeto Integrado Auto-ajuda nas Alterações da Pressão Arterial - CNPq 520445/97; ${ }^{2}$ Enfermeira, Mestre em Enfermagem; ${ }^{3}$ Doutor em Enfermagem, Professor Adjunto, Coordenadora do Projeto Integrado Auto-ajuda nas Alterações da Pressão Arterial - CNPq 520445/ 97, e-mail: thelma@ufc.br; ${ }^{4}$ Enfermeira do Hospital de Messejana, Docente da Universidade de Fortaleza, Mestre em Enfermagem. Universidade Federal do Ceará
} 


\section{INTRODUÇÃO}

A Hipertensão Arterial Sistêmica (HAS) é uma doença de alta prevalência em nosso país, atingindo cerca de $20 \%$ da população adulta jovem e $50 \%$ da população idosa. Dos casos de hipertensão, aproximadamente $90 \%$ são do tipo primário, cuja etiologia é desconhecida. Se não detectada precocemente, a hipertensão poderá ocasionar uma série de distúrbios, levando a lesões de órgãos vitais como coração, cérebro e rins.

Diante dessa realidade, sentimos a relevância e a magnitude do problema, carente de intervenção precoce e efetiva, como forma de evitar transtornos de maior gravidade, ao exemplo de acidente vascular cerebral, insuficiência coronariana, insuficiência renal e fenômenos ateroscleróticos, dentre outros. Em decorrência da análise sumariamente efetuada sobre a hipertensão, torna-se possível enquadrá-la como um grave problema de saúde em nosso meio, ao ponto de se constituir um verdadeiro desafio científico. É de se esperar que o enfermeiro, por desempenhar um importante papel dentro da equipe multiprofissional, mantenha-se permanentemente alerta quanto a essa situação, inclusive para resolver questões emergenciais.

Ao observarmos o idoso portador de uma doença crônica, como é o caso da hipertensão arterial sistêmica, encontramos, com freqüência, dificuldades na adaptação, o que, potencialmente, poderá gerar conflitos pessoais e familiares.

As doenças crônicas são caracterizadas pelo longo tempo de tratamento e pela limitação no estilo de vida, não só do portador, mas também de outros membros da família ${ }^{(1)}$.

Para haver um controle adequado da hipertensão arterial, não bastam apenas medidas de orientação; é preciso, também, desenvolver estratégias que auxiliem o indivíduo na mudança de atitudes contributivas para o controle da doença. As medidas de educação devem ser contínuas, visto que várias são as causas da não adesão ao tratamento, sendo uma delas a falta de motivação, podendo estar associada, principalmente, a fatores externos, como carência de sistema de apoio, dificuldades financeiras e de acesso ao sistema de saúde ${ }^{(2)}$.

Um dos grandes desafios para o portador da hipertensão é aceitar a convivência com o caráter crônico da enfermidade. A aceitação está, muitas vezes, associada a sentimentos de tristeza, raiva, agressividade e hostilidade, cuja superação só se faz através da conscientização do problema, com adesão ao tratamento proposto, tornando possível uma melhor adaptação à sua condição de saúde. Essa adaptação requer do indivíduo conhecimento relativo à doença, manifestações, sinais e sintomas, além de muita vontade de cooperar ativamente no tratamento ${ }^{(3)}$.

Dentro desse contexto, merecem especial enfoque as mudanças no estilo de vida, tais como: redução do peso corporal e da ingestão de sal, diminuição do consumo de álcool, além de prática regular de exercícios físicos e a não-utilização de drogas; essas medidas nem sempre são bem aceitas, causando constrangimentos e até mesmo resistência para a adesão. Torna-se imprescindível, então, o desenvolvimento de estratégias para o controle do problema, capazes de contribuir para uma melhor qualidade de vida e longevidade.

Os inúmeros problemas que afetam a qualidade de vida dos idosos, em um país em desenvolvimento, demandam, por sua vez, respostas urgentes em diversas áreas $^{(4)}$. Sendo assim, consideramos de extrema importância, para essa clientela, ações de caráter mais preventivo, principalmente no controle de doenças crônicas, como a hipertensão arterial.

Com base em estudo anterior, quando nos propusemos a realizar consultas de enfermagem com idosos, podemos relatar que, dentre aqueles que apresentaram diagnóstico confirmado de hipertensão e participavam, na ocasião, de programas específicos, poucos tiveram um controle desejável dos níveis de pressão arterial. A situação em causa veio nos trazer uma série de inquietações, dentre as quais destacamos: -será que estão se adaptando à nova mudança no estilo de vida? -que mecanismos de enfrentamento utilizam ${ }^{(5)}$.

Conforme comentamos anteriormente, o quadro de hipertensão, com freqüência, faz-se acompanhar de transtornos diversos para o cliente e/ou família, podendo gerar respostas ineficazes que impedem ou dificultam a adaptação. Essas respostas, por sua vez, poderão contribuir para a não adesão ao tratamento e, conseqüentemente, para possíveis complicações. No âmbito da questão, o enfermeiro poderá intervir, mostrando formas alternativas para amenizar tais problemas.

A hipertensão, por ser definida como uma doença 
multifatorial, é capaz de produzir inúmeros estímulos e, conseqüentemente, gerar respostas comportamentais das mais variadas ao portador, principalmente se ele for idoso. Percebemos, com isso, ser de extrema validade a aplicação do modelo teórico de Roy no planejamento e implementação da assistência, visto que, durante o tratamento da doença, é imprescindível a participação efetiva do cliente e a utilização de mecanismos de compensação aos estímulos que recebe.

Por ser a adaptação o foco de nosso estudo, consideramos que, para sua fundamentação teórica, o modelo conceitual avaliado seria o mais adequado.

Referido modelo de adaptação vislumbra os seres humanos, de forma individual e coletiva, como sistema holístico e adaptativo. O "Input", em forma de estímulo do meio interno e externo, ativa mecanismos de enfrentamento que agem para manter adaptação relativa aos quatro modos adaptativos (fisiológico, autoconceito, desempenho de papéis e interdependência). O resultado é observado através de respostas comportamentais, identificadas como adaptativas ou ineficazes. O comportamento adaptativo promove a integridade do sistema adaptativo, humano em termos de metas de sobrevivência, crescimento, reprodução e controle; já o comportamento ineficaz interrompe essa integridade ou não contribui para ela ${ }^{(6)}$.

Considerando a possibilidade de ser promovida uma melhor adaptação do idoso portador de hipertensão arterial à terapêutica, enfatizamos, por oportuno, a importância da aplicação do processo de enfermagem ${ }^{(6)}$ : 1. Avaliação de comportamentos - reunião de dados sobre o comportamento do sistema adaptativo humano e o corrente estado de adaptação; 2. Avaliação de estímulos - identificação de estímulos internos e externos que estão influenciando os comportamentos; 3 . Diagnóstico - formulação de classificações que interpretam os dados; 4. Estabelecimento de metas envolve a declaração de resultados comportamentais do cuidado de enfermagem que irão promover o processo adaptativo; 5. Intervenção - descrita como a seleção de ações de enfermagem para promover adaptação, mudando os estímulos ou fortalecendo o processo adaptativo; 6. Evolução - julgamento da eficácia da intervenção de enfermagem, em relação ao comportamento do sistema humano.

Em decorrência dos problemas levantados anteriormente, estabelecemos os seguintes objetivos: avaliar o processo adaptativo de um idoso portador de hipertensão arterial; identificar os diagnósticos de enfermagem e implementar ações de enfermagem.

\section{METODOLOGIA}

O trabalho foi estruturado na abordagem estudo de caso, correspondendo a: "uma caracterização abrangente para designar uma diversidade de pesquisas que coletam e registram dados de um caso particular ou de vários casos a fim de organizar um relatório ordenado e crítico de uma experiência, ou avaliá-la analiticamente, objetivando tomar decisões a seu respeito ou propor uma ação transformadora"(7).

Seu desenvolvimento contou com a participação de um idoso, membro de um grupo de auto-ajuda, atendido por um dos Projetos de Extensão do Departamento de Enfermagem da Universidade Federal de Fortaleza. As atividades de extensão incluíam, entre outras, a avaliação da pressão arterial e o acompanhamento dos clientes com valores alterados, inscritos ou não em programas de tratamento. $O$ trabalho possibilitou a identificação do cliente que era portador de hipertensão arterial e apresentava níveis de pressão sempre elevados, mesmo fazendo parte de um programa específico para o tratamento da doença. Além disso, mereceu uma atenção especial, por apresentar alguns fatores que poderiam aumentar o risco de morbimortalidade cardiovascular, tais como: sedentarismo, antecedentes familiares para doenças cardiovasculares e dificuldade em seguir o tratamento, principalmente $\mathrm{o}$ medicamentoso, relatando esquecimento de fazê-lo em alguns momentos. A isso estava aliado o fato de o idoso ser portador de diabetes mellitus e haver sofrido infarto do miocárdio há alguns meses. Diante dessa realidade, foi justificada a escolha do cliente.

O estudo foi realizado no período de outubro a novembro de 1999, em quatro visitas domiciliares. $\mathrm{Na}$ primeira visita, fizemos o levantamento de dados (levantamento de comportamentos e estímulos); na segunda, realizamos a confirmação de dados junto ao cliente e sua família; na terceira, confirmamos os diagnósticos estabelecidos e implementamos as devidas ações de enfermagem e, por fim, na quarta visita, fizemos a avaliação das intervenções. 
A coleta de dados foi do tipo primária, realizada junto ao cliente e família, mediante a utilização de um histórico, baseado no modelo teórico escolhido ${ }^{(8)}$, contemplando, portanto, os quatro modos adaptativos: fisiológico, autoconceito, desempenho de papéis e interdependência. Além disso, foram acrescentadas questões relativas à identificação de fatores de risco para doenças cardiovasculares.

A partir dessa coleta, avaliamos os comportamentos e seus estímulos, elaboramos diagnósticos de enfermagem e estabelecemos metas e intervenções para uma posterior avaliação, levando em consideração o processo de enfermagem adotado ${ }^{(6)}$.

Para elaboração dos diagnósticos do presente estudo, utilizamos o raciocínio diagnóstico composto de quatro fases: processamento de dados, formulação da declaração do diagnóstico, confirmação e documentação ${ }^{(9)}$.

Atendendo às exigências formais contidas na Resolução 196/96, sobre pesquisas que envolvem seres humanos, do Conselho Nacional de Saúde/Ministério da Saúde ${ }^{(12)}$, o estudo foi avaliado e aprovado por um Comitê de Ética. O cliente confirmou o seu aceite, mediante assinatura de termo de consentimento, sendo-lhe assegurado sigilo de identidade.

\section{RESULTADOS E DISCUSSÃO}

Avaliação de comportamentos e estímulos

A identificação de comportamentos e estímulos direciona as ações de enfermagem, de maneira a englobar o indivíduo que recebe o cuidado, considerandoo como ser integral em constante inter-relação com o meio interno e externo ${ }^{(10)}$.

Após o levantamento de dados, fizemos a avaliação de comportamentos e estímulos, considerando os modos adaptativos ${ }^{(6)}$, conforme demonstra a tabela a seguir:

Tabela 1 - Comportamentos ineficazes levantados e seus estímulos focais, contextuais e residuais, de acordo com o modelo adaptativo de Roy. Fortaleza, 1999

\begin{tabular}{|c|c|c|}
\hline Modos Adaptativos & Comportamentos & Estímulos - Focais (F), Contextuais (C) e Residuais (R) \\
\hline \multirow[t]{3}{*}{ Fisiológico } & 1. Limitaçẫo nas atividades da vida diária & $\begin{array}{l}\text { - Parestesia nos membros superior e inferior esquerdo (F) } \\
\text { - Hipertensấo (C) } \\
\text { - Infarto (C) } \\
\text { - AVC (C) }\end{array}$ \\
\hline & 2. Baixa acuidade visual & $\begin{array}{l}\text { - Uso de correçấo óptica inadequada (óculos)-(F) } \\
\text { - Envelhecimento (C) }\end{array}$ \\
\hline & 3. Dificuldade em conciliar o sono noturno & $\begin{array}{l}\text { - Dorme durante o dia (F) } \\
\text { - Falta de ocupaçẫo durante o dia (F) } \\
\text { - Envelhecimento (C) }\end{array}$ \\
\hline $\begin{array}{l}\text { Autoconceito e Desempenho } \\
\text { cle papéis }\end{array}$ & $\begin{array}{l}\text { 4. Vontade de voltar a ser como antes } \\
5 \text {. Sentimento de impotência diante dos } \\
\text { amigos } \\
\text { 6. Alteraçốes de papéis no relacionamento } \\
\text { doméstico e social }\end{array}$ & $\begin{array}{l}\text { - Situaçẫo de dependência (F) } \\
\text { - Déficit visual (F) } \\
\text { - Hipertensấo (C) } \\
\text { - Infarto (C) } \\
\text { - Cirurgia Cardiaca (C) } \\
\text { - AVC (C) } \\
\text { - Conceitos pessoais relativos aoprocesso saúde-doença(R) }\end{array}$ \\
\hline Interdependência & $\begin{array}{l}\text { 6. Sentimentos de solidẫo, em alguns } \\
\text { momentos }\end{array}$ & $\begin{array}{l}\text { - Impossibilidade de permanência ao lado do idoso pelas } \\
\text { pessoas que cuidam dele( }(F) \\
\text { - Mudança no estilo de vida }(C) \\
\text { - Necessidade gregária }(R)\end{array}$ \\
\hline
\end{tabular}

No modelo adotado $^{(6)}$, o comportamento é definido como uma ação ou reação a circunstâncias específicas, podendo ser observável ou não. Com referência a esse caso, em particular, percebemos diversas reações à situação de conflito inicial, ou seja, a hipertensão que gerou complicações posteriores, culminando em um infarto, seguido de uma cirurgia cardíaca e de um acidente vascular cerebral.

$O$ fato em si produziu reflexos na mudança do estilo de vida, com conseqüente elaboração de respostas ineficazes, tais como: limitação nas atividades da vida diária; sentimento de impotência diante dos amigos; alterações no desempenho de papéis e, por fim, 
sentimentos de solidão, apresentados em alguns momentos.

Para cada comportamento, tivemos vários estímulos, demonstrando a dinâmica do sistema adaptativo humano. O estímulo é definido como aquilo que provoca uma resposta no indivíduo, podendo ser de natureza focal: estímulo interno ou externo que confronta imediatamente o sistema adaptativo humano; contextual: os outros estímulos internos ou externos evidentes na situação e que contribuem diretamente para o efeito do estímulo focal; e residual: definido como aquele que tem efeito indeterminado no comportamento do sistema adaptativo humano ${ }^{(6)}$.

Vale ressaltar a predominância de estímulos focais, seguidos dos contextuais e residuais. Diante disso, tivemos como estímulos focais: parestesia nos membros, correção óptica inadequada, sono e falta de ocupação durante o dia; situação de dependência; déficit visual e impossibilidade de permanência, ao seu lado, das pessoas que lhe prestam cuidados; contextuais: hipertensão, infarto, cirurgia cardíaca, acidente vascular cerebral, envelhecimento e mudança no estilo de vida; residuais: conceitos pessoais relativos ao processo saúde-doença e necessidade gregária.

É notável a pequena ocorrência de identificação dos estímulos residuais, talvez por envolver crenças e valores, dificultando, assim, a nossa percepção a seu respeito.

Diagnósticos, metas e intervenções de enfermagem

Tabela 2 - Estabelecimento de diagnósticos e intervenções de enfermagem. Fortaleza, 1999

\begin{tabular}{|c|c|}
\hline Diagnósticos & Intervençöes \\
\hline 1. Mobilidade, andar elou coordenaçấo restritos & $\begin{array}{l}\text { - Incentivar a continuidade nas sessốes de fisioterapia } \\
\text { - Ensinar a realizar seqüência de movimentos com os membros afetados e nấo afetados } \\
\text { - Encorajar a deambulaçấo em caminhadas curtase freqüentes } \\
\text { - Encorajar a pessoa a usar o braço afetado nas atividades de autocuidado }\end{array}$ \\
\hline 2. Deficiência de um sentido primário & $\begin{array}{l}\text { - Estimular o cliente a procurar um serviço de oftalmologia } \\
\text { - Orientar a familia para proporcionar um ambiente seguro e bem iluminado (evitar objetos } \\
\text { que possamprovocar quedas e fraturas) } \\
\text { - Orientar a familia para evitar mudar o ambiente físico com freqüência } \\
\text { - Incentivar a pessoa a solicitar auxilio na deambulaçäo noturna } \\
\text { - Instruir a pessoa a usar sapatos bem ajustados, com solado antiderrapante }\end{array}$ \\
\hline 3. Privaçẫo do sono & $\begin{array}{l}\text { - Estabelecer com a pessoa um horário para um programade atividades diumas (carnirhada, } \\
\text { fisioterapia...) } \\
\text { - Orientar o cliente para limitar o sono durante o dia } \\
\text { - Orientar o cliente para diminuir a ingesta de bebidas com cafeína } \\
\text { - Orientar para atividades de relaxamento }\end{array}$ \\
\hline 4. Baixa auto-estima & $\begin{array}{l}\text { - Encorajar a pessoa a expressar os sentimentos, especialmente quanto à maneira como } \\
\text { se sente, pensa ou vê a si própria } \\
\text { - Encorajar apessoa a fazer pergurtas sobreo problema de saide, otratamento, oprogresso } \\
\text { e o prognóstico } \\
\text { - Encorajar as opçốes para a soluçẫo de problemas }\end{array}$ \\
\hline 5. Transiçẫo de papel & $\begin{array}{l}\text { - Estimular o desenvolvimento das atividades anteriores (contexto farniliar) } \\
\text { - Mostrar sua importância no contexto social e familiar } \\
\text { - Estimular o desenvolvimento da autonomia } \\
\text { - Orientar a familia para evitar a superproteçẫo } \\
\text { - Incentivar o retorno ao grupo de apoio (Grupo vida) }\end{array}$ \\
\hline 6. Solidẫo & $\begin{array}{l}\text { - Apoiar as defesas saudáveis } \\
\text { - Incentivar a relaçấo com os outros } \\
\text { - Auxiliar a identificar os cursos alternativos de açẫo } \\
\text { - Proporcionar momentos para discussấo de sentimentos } \\
\text { - Incentivar o retorno ao grupo de apoio (Grupo Vida) }\end{array}$ \\
\hline
\end{tabular}

Finda a etapa de avaliação de comportamentos e estímulos, partimos para a elaboração de diagnósticos de enfermagem, utilizando a tipologia diagnóstica do modelo teórico $^{(6)}$.
Uma vez estabelecidos os diagnósticos, fizemos a confirmação com o cliente, tendo como resultado alterações comportamentais nos quatro modos adaptativos, contemplando um total de seis diagnósticos, 
conforme se segue: 1. mobilidade, andar e/ou coordenação restritos; 2. deficiência de um sentido primário; 3. privação do sono; 4. auto-estima baixa; 5 . transição de papel e 6 . solidão.

Observa-se que os diagnósticos foram relacionados, principalmente, às alterações orgânicas acarretadas pelo envelhecimento e pela doença crônica (hipertensão). Além disso, a mudança no estilo de vida trouxe implicações no modo de autoconceito, desempenho de papéis e interdependência.

O modelo teórico ${ }^{(6)}$, colocando os seres humanos como um sistema holístico e adaptativo em constante interação com o meio, levou-nos a perceber, em manifestação do próprio cliente, uma necessidade de adaptação às mudanças na estrutura de vida, como forma de encontrar alternativas capazes de amenizar as perdas, sejam elas biológicas, psicológicas, econômicas ou espirituais.

A partir dos diagnósticos estabelecidos, fizemos um planejamento das metas e intervenções de enfermagem, na tentativa de oferecer uma contribuição ao processo adaptativo do cliente, permitindo-se, com isso, a substituição de respostas ineficazes por respostas adaptativas, visando, assim, a uma melhor qualidade de vida e, conseqüentemente, a uma maior longevidade. As intervenções para os diagnósticos identificados tiveram, como fundamento, sugestões da literatura específica ${ }^{(11)}$ e o conhecimento enfermagem geriátrica que as autoras do estudo possuem.

Nesse âmbito, estabelecemos as metas de enfermagem a seguir relacionadas:

- promover uma mobilidade física adequada;

- amenizar o déficit visual;

- melhorar o padrão de sono;

- elevar o autoconceito;

- incentivar a execução de atividades ocupacionais;

- promover uma melhor integração com os outros.

As metas de enfermagem buscam restabelecer e/ou manter o equilíbrio, a execução de atividades ocupacionais, sendo possível relacioná-las às intervenções necessárias ${ }^{(10)}$.

É válido colocarmos em evidência que as intervenções foram elaboradas, principalmente, com base nos estímulos focais, em virtude de estarem confrontando, diretamente, o cliente e por serem, no presente caso, mais passíveis de resolutividade. Sabemos, porém, da importância de trabalharmos com os estímulos contextuais e residuais, levando em conta que a mudança de comportamento não se dá apenas intervindo sobre os focais.

Avaliação das intervenções de enfermagem

Através da realização de quatro visitas domiciliares, tivemos a oportunidade de implementar as ações de enfermagem propostas, assim como avaliar o nível de respostas comportamentais do cliente, diante das intervenções estabelecidas.

Observamos, desde a primeira visita, modificações adaptativas significantes, provavelmente favorecidas pelo clima de empatia estabelecido em momento anterior, tendo em vista que o idoso participava de um grupo de auto-ajuda para a terceira idade, denominado "Grupo Vida", do qual também fazemos parte.

Em consideração às intervenções de enfermagem, podemos relatar que essas foram importantes no alcance das metas, já que permitiram, de maneira geral, a promoção de comportamentos adaptativos, tendo, como conseqüência, a superação de algumas repostas ineficazes. Isso ficou bastante claro, nas seguintes constatações:

- o cliente relatou melhora na mobilidade física, uma vez que está participando efetivamente das sessões de fisioterapia (três vezes na semana), bem como realizando caminhadas (três vezes na semana, durante 30 minutos) e exercícios de movimentação dos membros;

- no que se refere ao déficit visual, o cliente procurou um serviço de oftalmologia, sendo prescrita correção óptica adequada, a ser providenciada posteriormente, em virtude da falta de condição financeira para aquisição imediata de lentes;

- na reavaliação do padrão 1 de sono, há uma melhora gradativa, uma vez que está ocupando melhor o dia (fisioterapia, caminhadas) e limitando o sono diurno. No entanto, não está conseguindo reduzir a ingesta de café, segundo relato da esposa;

- em relação ao distúrbio no autoconceito e interação social prejudicada, percebemos, também, um bom nível de evolução, em decorrência de uma melhor expressão de sentimentos; desenvolvimento de atividades da vida diária, de forma bem mais independente; diminuição da superproteção por parte da esposa, permitindo que o cliente se dirija ao Centro de Fisioterapia apenas 
acompanhado por um amigo que está fazendo sessões no mesmo centro, estabelecendo mais um laço de amizade; reconhecimento de sua importância no contexto social e familiar; restabelecimento de sua autonomia e vontade expressa de retornar ao grupo de apoio (Grupo Vida).

Diante das considerações feitas em relação ao processo adaptativo do cliente às situações geradoras de conflitos, merece destaque a participação contínua da esposa. Sendo assim, alguns modos adaptativos, por se fazerem presentes no âmbito interno do indivíduo, precisam de um trabalho mais cauteloso, com a participação de outras pessoas, sobretudo de familiares $^{(10)}$.

\section{CONSIDERAÇÕES FINAIS}

As reflexões feitas, com base no estudo, permitiram as seguintes inferências:

\section{REFERÊNCIAS BIBLIOGRÁFICAS}

1. AraújoTL, Maciel ICF, Maciel GGF, Silva ZMSA. Reflexo da hipertensão arterial no sistema familiar. Rev Soc Cardiol Estado de São Paulo 1998; 2(Supl A):1-6.

2. Pessuto J, Carvalho EC. Fatores de risco em indivíduos com hipertensão arterial. Rev Latino-am Enfermagem 1998 janeiro; 6(1):33-9.

3. Pierin AMG, Car MR. Instrumento de consulta de enfermagem a pessoas com hipertensão arterial em tratamento ambulatorial. Rev Esc Enfermagem USP 1992 março; 26(1):17-32.

4. Assis M. O envelhecimento e suas conseqüências. In: Caldas CP.A saúde do idoso: a arte de cuidar. Rio de Janeiro (RJ): UERJ; 1998. p.39-48.

5. Oliveira TC. O processo de envelhecimento e sua influência na saúde cardiovascular e ocular. [monografia]. Fortaleza (CE): Departamento de Enfermagem/UFC; 1999.

6 . Roy SC, Andrews HA. The nursing process according to the Roy adaptation model. In: Roy Sc, Andrews HA. The Roy adaptation model. Stamford: Appleton \& Lange; 1999. p.6396.

7. Chizzotti A. Pesquisa em ciências humanas e sociais. In: Chizzotti A. Pesquisa qualitativa. São Paulo (SP): Cortez; 1991. p.77-104.

8. Christensen PJ. Assessment data collection to the family client. In: Christensen PJ, Kenney JW. Nursing process: application of conceptual models. St. Louis: Mosby; 1995. p. 70-7.

9. Iyer PW, Taptich, BJ, Bernocchi-Losey D. O processo diagnóstico. In: lyer PW, Taptich BJ, Bernocchi-Losey D. Processo e diagnóstico em enfermagem. Porto Alegre: Artes Médicas; 1993. p. 57-72.

10. Lopes MVO, Araujo TL. Utilização da teoria de Roy e da
- a aplicação do modelo teórico ${ }^{(6)}$ foi extremamente importante para o presente caso, haja vista ter proporcionado suporte para melhor adaptação do cliente a uma situação de conflito, gerada, de início, pela doença crônica e transtornos decorrentes (infarto e acidente vascular cerebral);

- através do estabelecimento de metas e intervenções de enfermagem, frente aos diagnósticos elaborados, observamos a substituição de respostas ineficazes por respostas adaptativas. A situação em referência é extremamente satisfatória para o cliente que, apesar de se encontrar em condição estável, em relação ao quadro da hipertensão arterial sistêmica, merece uma atenção especial, em virtude das complicações apresentadas em momento anterior;

- o cliente apresentou uma boa apreensão, no que se refere às intervenções estabelecidas, apresentando-se mais seguro e autoconfiante, repercutindo em melhora no autoconceito, desempenho de papéis e interdependência.

linguagem dos diagnósticos de enfermagem: estudo de caso de um paciente com problemas cardíacos. Rev Mineira Enfermagem 1998 julho/dezembro; 2(2):104-10.

11. Carpenito LJ. Manual de diagnóstico de enfermagem. $6^{a}$ ed. Porto Alegre (RS): Artes Médicas; 1999.

12. Ministério da Saúde (BR). Diretrizes e normas regulamentadoras de pesquisa envolvendo seres humanos. Brasília (DF): Ministério da Saúde; 1996. 